\title{
A New Portfolio Rebalancing Model with Transaction Costs
}

\author{
Meihua Wang, ${ }^{1}$ Cheng Li, ${ }^{1}$ Honggang Xue, ${ }^{1}$ and Fengmin $\mathrm{Xu}^{2}$ \\ ${ }^{1}$ School of Finance and Economics, Xi'an Jiaotong University, Xian 710061, China \\ ${ }^{2}$ School of Mathematics and Statistics, Xian Jiaotong University, Xian 710049, China \\ Correspondence should be addressed to Fengmin Xu; fengminxu@mail.xjtu.edu.cn
}

Received 4 December 2013; Revised 1 March 2014; Accepted 4 March 2014; Published 28 April 2014

Academic Editor: Farhad Hosseinzadeh Lotfi

Copyright (C) 2014 Meihua Wang et al. This is an open access article distributed under the Creative Commons Attribution License, which permits unrestricted use, distribution, and reproduction in any medium, provided the original work is properly cited.

\begin{abstract}
A portfolio rebalancing model with self-finance strategy and consideration of $\mathrm{V}$-shaped transaction cost is presented in this paper. Our main contribution is that a new constraint is introduced to confirm that the rebalance necessity of the existing portfolio needs to be adjusted. The constraint is constructed by considering both the transaction amount and transaction cost without any additional supply to the investment amount. The V-shaped transaction cost function is used to calculate the transaction cost of the portfolio, and conditional value at risk ( $\mathrm{CVaR})$ is used to measure the risk of the portfolios. Computational tests on practical financial data show that the proposed model is effective and the rebalanced portfolio increases the expected return of the portfolio and reduces the CVaR risk of the portfolio.
\end{abstract}

\section{Introduction}

During the past six decades, many papers have been written on the theory and practice of portfolio selection with most researchers concentrating on the initial investment. However, with the elapse of time, the initial portfolio may become nonoptimal. If an investor hopes to hold the investment on the portfolio in the following periods, it is necessary to adjust the portfolio based on either maximizing expected return of the portfolio or minimizing the risk of the portfolio. This is the so-called portfolio rebalancing (revision, adjusting) problem [1].

Our main concern is self-finance portfolio rebalancing problem which means that the investor will not supply any additional investment amount. Most researchers focus on the modeling and algorithm design based on various transaction cost functions. Jouini and Kallal [2] establish the theory with consideration of transaction costs based on martingale measures. Arnott and Wagner [3] find that ignoring transaction costs often results in an inefficient portfolio in practice. Yoshimoto [4] also reaches the same conclusion through empirical analysis. Konno and Yamamoto [5] aim to minimize the transaction costs associated with rebalance in the framework of mean absolute deviation with concave transaction cost function. Then, they obtain a concave minimization problem and propose a branch-and-bound method to get optimal rebalance amount. Lobo et al. $[6,7]$, Best and Hlouskova [8] propose a self-finance strategy with linear transaction costs. A self-finance constraint is included in the portfolio rebalancing model. The investor aims either to maximize the expected return of the resulting portfolio after paying transaction costs under a given tolerated level of risk and other constraints or to minimize the total rebalance transaction costs subject to a specified requirement on the expected return of the portfolio, risk, self-finance, and other constraints. Best and Hlouskova [9] consider the problem of maximizing as an expected utility function of $n$ assets with linear transaction costs and present a method for solving the $3 n$-dimensional problem based on optimality conditions for the higher-dimensional problem. The new method is compared to the barrier method implemented in Cplex in a series of numerical experiments and outperforms the barrier method by a larger and larger factor as the size of the transaction cost increases. Mitchell and Braun [10] extend the standard portfolio selection problem to consider convex transaction costs incurred when rebalancing an investment portfolio. They suggest rescaling by the funds available after paying transaction costs and then obtain a fractional 
programming problem, which can be reformulated as an equivalent convex program of size comparable to the model without transaction costs. However, existing models do not consider whether the initial portfolio is worthwhile to adjust or not.

In the existing literature on portfolio rebalancing problem, variance $[6,8,11]$, absolute deviation [5], and entropic risk measure [12] are usually used to measure the risk of the portfolio. In recent years, shortfall measure has become so popular and practical in the risk management area. Value at risk (VaR) is an important shortfall risk measurement, but it has some undesirable mathematical characteristics such as lack of subadditivity and convexity [13]. VaR is not coherent except the case when the return rate of portfolios is of normal distribution. To overcome these shortcomings, recent research focuses on coherent risk measurement, especially on CVaR. Tyrrell Rockafellar and Uryasex [14] propose the use of CVaR in portfolio selection problems. The main advantage of CVaR is that whether CVaR is used to be an objective function or a constraint in portfolio selection problems; the resulting model can be converted into a linear programming problem based on discrete samples [15]. Furthermore, there is also an equivalent linear programming if one deals with continuous problems [16].

Our main contribution is adding a constraint to confirm the necessity of rebalance. The portfolio rebalancing problem with self-finance strategy is modified by introducing a constraint of expected excess return requirement in this paper. CVaR is used to measure the risk of portfolios. Transaction cost is one of the main factors for an investor to take into account in adjusting the existing portfolio. It is commonly assumed in literature that the transaction cost is a $\mathrm{V}$-shaped function of the transaction amount and then we accept this. However, the proposed model can be easily extended to handle more complex transaction cost functions. The expected excess return on portfolios is specified by the investor and when the expected excess return is satisfied, the rebalancing on the initial portfolio will be performed. One of the main contributions of this paper is to construct the return requirement constraint to confirm the necessity of rebalance.

The rest of the paper is organized as follows. The new model with self-finance strategy is presented in Section 2 where the constraint for expected excess return of portfolios will be given. Computational tests are stated in Section 3. Conclusion and future researches are given in Section 4.

Throughout the paper, prime $\left(^{\prime}\right)$ denotes transposition of vectors without special declaration. The notation $s_{i}$ or $(s)_{i}$ is used to denote the $i$ th component of the vector $s$.

\section{A New Portfolio Rebalancing Model}

The portfolio rebalancing model with self-finance strategy and an excess return requirement will be presented in this section. It is assumed that the investor holds a portfolio which is optimal at the beginning of a previous period. However, with the elapse of time, the initial portfolio needs to be evaluated whether it is still optimal or not, due to changes of conditions in financial markets or disclosure of much more information. If it becomes nonoptimal, rebalancing procedure will be carried out on the portfolio, without providing any additional supply to pay for the transaction costs. That means that paying for transaction costs will come from the capital of selling some assets. The rebalancing goal in [7] is to maximize the expected return of the portfolio subject to some practical constraints. Different from [7], the rebalancing goal of this paper is to minimize the CVaR risk of the portfolio subject to some practical constraints including specified excess return, when the initial portfolio needs to be adjusted. The problem whether the portfolio needs to be adjusted will be controlled by the constraint: the expected excess return should be satisfied. Only when this is true, the portfolio rebalancing procedure is worthwhile to be performed.

2.1. The Requirement of Excess Return. Assume that an investor holds a portfolio consisting of $n$ assets and $s=$ $\left(s_{1}, s_{2}, \ldots, s_{n}\right)^{\prime}$ is used to denote the initial portfolio, where $s_{i}$ denotes the amount (such as dollars) invested on the $i$ th asset. Let $r=\left(r_{1}, r_{2}, \ldots, r_{n}\right)^{\prime}$ be the return rates of the assets, with $\bar{r}=\left(\bar{r}_{1}, \bar{r}_{2}, \ldots, \bar{r}_{n}\right)^{\prime}$ being the expected return rate of the given period. Let $x=\left(x_{1}, x_{2}, \ldots, x_{n}\right)^{\prime}$ be the amount transacted in all assets, with $x_{i}>0$ for buying and $x_{i}<0$ for selling. After transacting, the resulting portfolio is $s+x$. The expected return from the adjusting is

$$
\bar{r}^{\prime} x
$$

The transaction cost associated with transaction amount $x$ is given by

$$
C(x)=\sum_{i=1}^{n} C_{i}\left(x_{i}\right),
$$

where $C_{i}\left(x_{i}\right)$ is the transaction cost on the $i$ th asset when $x_{i}$ amount is traded. Hence, the expected excess return from the adjusting is

$$
\bar{r}^{\prime} x-C(x) .
$$

It can be seen that the transaction costs associated with purchasing or selling have a significant effect in portfolio rebalancing problem.

Let $\alpha>0$ be an additional excess return requirement given by the investor. Then, the constraint controlling the portfolio rebalancing can be expressed as

$$
\bar{r}^{\prime} x-C(x) \geq \alpha .
$$

It can be seen from (4) that if there exists no feasible solution $x$ to satisfy (4), the rebalancing on the portfolio is not necessary and the investor can still hold the portfolio. Hence, the new portfolio rebalancing model is expressed as follows:

$$
\begin{array}{ll}
\min & f(s+x) \\
\text { s.t. } & \bar{r}^{\prime} x-C(x) \geq \alpha \\
& x \in \mathbf{M},
\end{array}
$$


where $f(s+x)$ is a risk measure of the portfolio $s+x$ and $\mathbf{M}$ is the set of feasible portfolios restricted by some practical constraints which will be discussed in the following subsections.

2.2. The CVaR Risk of Portfolios. It is known that CVaR is a coherent risk measure and becomes more and more popular in practical risk management area. Moreover, no matter that the CVaR is used as an objective function or a constraint, the resulting portfolio rebalancing model can be converted into a linear programming problem.

Let $l(x, r)$ be the loss function associated with the decision vector $x$ and the return vector $r$ of portfolios. Let $p(r)$ be the density function of the return vector $r$. Then, the probability of $l(x, r)$ not exceeding a threshold $w$ is given by

$$
\psi(x, w)=\int_{l(x, r) \leq w} p(r) d r .
$$

Definition 1 ((VaR) [14]). The VaR risk of the loss associated with a decision vector $x$ and a specified probability level $\theta \in$ $(0,1)$ is the value

$$
\operatorname{VaR}_{\theta}(x)=\min \{w \in \mathbf{R}: \psi(x, w) \geq \theta\} .
$$

Definition 2 ((CVaR) [14]). The CVaR risk of the loss associated with a decision vector $x$ and a specified probability level $\theta \in(0,1)$ is given by

$$
\operatorname{CVaR}_{\theta}(x)=\frac{1}{1-\theta} \int_{l(x, r) \geq \operatorname{VaR}_{\theta}(x)} l(x, r) p(r) d r .
$$

Substituting (7) into the problem $(P)$ generates

$$
\begin{array}{ll}
\min _{x} & \frac{1}{1-\theta} \int_{l(x, r) \geq \operatorname{VaR}_{\theta}(x)} l(x, r) p(r) d r \\
\text { s.t. } & \bar{r}^{\prime} x-C(x) \geq \alpha \\
& x \in \mathbf{M} .
\end{array}
$$

Since the value of $\operatorname{VaR}_{\theta}(x)$ in the problem is unknown, it is difficult to solve the problem. Tyrrell Rockafellar and Uryasex in $[14,15]$ propose the following auxiliary function to replace the $\mathrm{CVaR}$ risk function:

$$
\begin{aligned}
F_{\theta}(x, w) & =w+\frac{1}{1-\theta} \mathbf{E}\left\{[l(x, r)-w]^{+}\right\} \\
& =w+\frac{1}{1-\theta} \int[l(x, r)-w]^{+} p(r) d r .
\end{aligned}
$$

Here, $\mathbf{E}\{\xi\}$ denotes the expectation of the random variable $\xi$ and $[l(x, r)-w]^{+}=\max \{l(x, r)-w, 0\}$.

Theorem 3 (see [14]). Minimizing CVaR of the loss associated with $x$ and $\theta$ over all $x \in \mathbf{X}$ is equivalent to minimizing $F_{\theta}(x, w)$ over all $(x, w) \in \mathbf{X} \times \mathbf{R}$, in the sense that

$$
\min _{x \in \mathbf{X}} \operatorname{CVaR}_{\theta}(x) \Longleftrightarrow \min _{(x, w) \in \mathbf{X} \times \mathbf{R}} F_{\theta}(x, w) .
$$

The theorem indicates that the solutions of both optimization problems are the same. When rebalancing is considered for a portfolio $s$, the loss function $l(x, r)=-(s+x)^{\prime} r$. Then, the objective function associated with a given probability $\theta \in(0,1)$ can be expressed as

$$
\begin{aligned}
\min _{(x, w) \in(\mathbf{X} \times \mathbf{R})} F_{\theta}(x, w) \\
\quad=\min _{(x, w) \in(\mathbf{X} \times \mathbf{R})} w+\frac{1}{1-\theta} \int\left[-(s+x)^{\prime} r-w\right]^{+} p(r) d r .
\end{aligned}
$$

The function $F_{\theta}(x, w)$ can further be approximated when the values of the return vector $r$ are given by a collection of discrete vectors $\left\{r^{1}, r^{2}, \ldots, r^{T}\right\}$, where $r^{t}$ is the return rate of assets at the subperiod $t$; then, a corresponding approximation to the function $F_{\theta}(x, w)$ is

$$
\widetilde{F}_{\theta}(x, w) \approx w+\frac{1}{(1-\theta) T} \sum_{t=1}^{T}\left[-(s+x)^{\prime} r^{t}-w\right]^{+} .
$$

Although the function $\widetilde{F}_{\theta}(x, w)$ is not differentiable, the resulting problem can be readily solved, either by line search techniques or by converting it into a linear programming problem.

2.3. The Self-Finance Constraint. The new model is established under the self-finance strategy. The self-finance constraint can be expressed by the total value of the initial portfolio equal to the sum of the value of the final portfolio and the transaction costs

$$
\mathbf{1}^{\prime}(s+x)+C(x)=\mathbf{1}^{\prime} s,
$$

where $\mathbf{1}$ is an $n$-dimensional row vector with all entries equal to one. Equation (13) can be simplified as

$$
\mathbf{1}^{\prime} x+C(x)=0 .
$$

This constraint says that the total transaction costs are obtained by selling assets more than buying assets.

2.4. Short Sale Constraints. Due to market regulations, short sale constraints should be considered. Short sale limitation in each asset is represented by

$$
s_{i}+x_{i} \geq-p_{i}, \quad i=1,2, \ldots, n,
$$

where $p_{i}$ is the maximum amount of short sale permitted for the $i$ th asset. If short sale is not permitted, $p_{i}$ is set to 0 .

2.5. Constraints for Share Restrictions. Due to investor's limited investment capability and diversification requirement, the investment amount or proportion on each asset has a lower bound and an upper bound. Consider

$$
l_{i} \leq s_{i}+x_{i} \leq u_{i}
$$


or

$$
L_{i} \leq \frac{s_{i}+x_{i}}{\sum_{i=1}^{n} s_{i}} \leq U_{i}
$$

where $l_{i}\left(\right.$ or $L_{i}$ ) and $u_{i}$ (or $U_{i}$ ) are the lower bound and the upper bound of the investment amount (or proportion) on the $i$ th asset after transacting. If short sale is not permitted, then $l_{i} \geq 0$, and short sale constraint (15) can be omitted.

With all of the above constraints, the new rebalance model is given as follows:

$$
\begin{array}{ll}
\min _{x, w} & w+\frac{1}{(1-\theta) T} \sum_{t=1}^{T}\left[-(s+x)^{\prime}\left\{r^{t}\right\}-w\right]^{+} \\
\text {s.t. } & \bar{r}^{\prime} x-C(x) \geq \alpha \\
& \mathbf{1}^{\prime} x+C(x)=0 \\
& l_{i} \leq s_{i}+x_{i} \leq u_{i}, \quad i=1,2, \ldots, n .
\end{array}
$$

Let $d_{i}^{B}\left(d_{i}^{S}\right)$ be the transaction cost ratio for buying or selling one unit amount of the $i$ th asset, $i=1,2, \ldots, n$. Then,

$$
C_{i}\left(x_{i}\right)= \begin{cases}d_{i}^{B} x_{i}, & \text { if } x_{i}>0 \\ -d_{i}^{S} x_{i}, & \text { if } x_{i}<0\end{cases}
$$

Variables $x_{i}^{+}$and $x_{i}^{-}, i=1,2, \ldots, n$, are introduced for convenience of calculation with $x_{i}^{+}=\max \left\{0, x_{i}\right\}$ and $x_{i}^{-}=$ $-\min \left\{0, x_{i}\right\}$. Then, $x_{i}^{+} \geq 0, x_{i}^{-} \geq 0$, for $i=1,2, \ldots, n$, and the transaction costs function $C_{i}\left(x_{i}\right)$ could be represented as

$$
C_{i}\left(x_{i}\right)=d_{i}^{B} x_{i}^{+}+d_{i}^{S} x_{i}^{-},
$$

where we have $x_{i}=x_{i}^{+}-x_{i}^{-}$.

Since there are $[\cdot]^{+}$representations in (18), the problem is not differentiable. Let

$$
q_{t}=\left[-(s+x)^{\prime} r^{t}-w\right]^{+}, \quad t=1,2, \ldots, T
$$

Then,

$$
q_{t} \geq-(s+x)^{\prime} r^{t}-w, \quad t=1,2, \ldots, T
$$

and the problem (18) can be converted into the following:

$$
\begin{array}{ll}
\min _{x^{+}, x^{-}, q, w} & w+\frac{1}{(1-\theta) T} \sum_{t=1}^{T} q_{t} \\
\text { s.t. } & \sum_{i=1}^{n} \bar{r}_{i}\left(x_{i}^{+}-x_{i}^{-}\right) \\
& -\sum_{i=1}^{n}\left(d_{i}^{B} x_{i}^{+}+d_{i}^{B} x_{i}^{-}\right) \geq \alpha \\
& q_{t} \geq-\left(s+x^{+}-x^{-}\right)^{\prime} r^{t}-w
\end{array}
$$

$$
\begin{aligned}
& \sum_{i=1}^{n}\left(x_{i}^{+}-x_{i}^{-}\right)+\sum_{i=1}^{n}\left(d_{i}^{B} x_{i}^{+}+d_{i}^{B} x_{i}^{-}\right)=0 \\
& l_{i} \leq s_{i}+x_{i}^{+}-x_{i}^{-} \leq u_{i} \\
& q_{t} \geq 0, \quad x_{i}^{+} \geq 0, \quad x_{i}^{-} \geq 0 \\
& i=1,2, \ldots, n ; \quad t=1,2, \ldots, T .
\end{aligned}
$$

Problem (23) is a linear programming and algorithms such as the simplex algorithm and interior point algorithms $[17,18]$ can be employed to find its solution.

\section{Computational Results}

In this section, we will test the proposed portfolio rebalancing model with practical data. Assume that the investor holds an existing portfolio which is an optimal portfolio invested one year ago. As time goes by, the return and the risk of the existing portfolio have changed and whether the portfolio is still optimal is unknown. The proposed rebalancing model will be used to help the investor to decide whether to adjust the portfolio or not.

3.1. Test Data Sets and Steps. The model will be tested on the data sets described in [19]. Beasley has built an OR-Library which is a publicly available collection of test data sets for a variety of operations research problems. A series of weekly closing prices from 1992 to 1994 for 15 component stocks of S\&P index are selected.

The data sets include 105 history weekly prices of the 15 selected component stocks, by which we could obtain 104 weekly return rates for each stock (it is assumed that there are 52 weeks per year). The time period for each week is denoted as $t=1,2, \ldots, 104$. An initial portfolio is constructed based on the first 52 return rates of the 15 stocks at time $t=52$. After one year later (at time 104), it is necessary to evaluate the optimality of the existing portfolio based on the last 52 return rates of these stocks. The test experiments will be performed in the following two stages.

Stage 1. The data sets are divided into two parts. The first 52 return rates (one year) are applied to construct the initial portfolio. The investor follows the initial portfolio to make investments on the selected stocks at time $t=52$.

Stage 2. After one year has passed (at time $t=104$ ), the proposed model will be used to evaluate whether the portfolio is still optimal or not by using the last 52 return rates. If it is not optimal, adjusting to the portfolio is necessary and the optimal adjusting strategy will be given by using the proposed rebalancing model.

All the computational tests are run on a personal computer with Pentium Pro $1794 \mathrm{MHZ}$ and $512 \mathrm{MB}$ memory.

3.2. The Initial Portfolio. Assume that the investor has the initial portfolio with a total wealth of $W$ dollar at time $t=52$. 
Transaction costs are not considered in constructing the initial portfolio so that the total amount of the portfolio is $W$. Yearly risk of $\mathrm{CVaR}$ is also used to measure the risk of the portfolio. The initial portfolio is constructed by minimizing the CVaR risk of the portfolio, subjecting to specified return rate. That is, the initial portfolio is constructed based on the first 52 weekly return rates of the selected 15 component stocks and it is the solution of the following portfolio selection model:

$$
\begin{array}{ll}
\min _{s} & \operatorname{CVaR}_{\theta}(s) \\
\text { s.t. } & \sum_{i=1}^{n} \bar{r}_{i} s_{i} \geq \lambda W \\
& \sum_{i=1}^{n} s_{i}=W \\
& \varepsilon_{i} W \leq s_{i} \leq \delta_{i} W \\
& i=1,2, \ldots, n,
\end{array}
$$

where $s=\left(s_{1}, s_{2}, \ldots, s_{n}\right)^{\prime}(n=15)$ is the initial portfolio, $s_{i}$ is the investment amount on the $i$ th stock at time $t=52, \bar{r}_{i}$ is yearly expected return rate of the $i$ th stock estimated from the data $r_{i}^{t}$ over time period $t=1$ to $t=52, \mathrm{CVaR}_{\theta}(s)$ is the CVaR risk of the portfolio $s$ with a specified confidence level $\theta \in(0,1), W$ is the total amount of the portfolio, $\lambda>0$ is the requirement of the expected return specified by the investor, and $\varepsilon_{i}$ and $\delta_{i}$ are the investment lower and upper bound scale limitations on the $i$ th stock. Note that the transaction cost to the initial portfolio is not considered; that is, the transaction cost will be supplied by the investor, so that the total amount of the initial portfolio is $W$.

Problem (24) could be converted into a linear programming by replacing $\mathrm{CVaR}_{\theta}(s)$ with the function

$$
\widetilde{F}_{\theta}(s, w) \approx w+\frac{1}{(1-\theta) T} \sum_{t=1}^{T}\left[-s^{\prime} r^{t}-w\right]^{+}
$$

where $T=52$. The "linprog" function in MATLAB is used to solve the resulting linear programming.

The following parameter values are used in the model to generate the initial optimal portfolio:

(1) confidence level $\theta=95 \%$;

(2) initial wealth $W=\$ 100000000$;

(3) $\lambda=10 \%$ (the maximal yearly return rate among the 15 stocks is $27.66 \%$, and the minimal one is $-14.25 \%$ );

(4) $d_{i}=0.002$ for $i=1,2, \ldots, n$ (transaction cost per unit amount investment);

(5) lower bound $\varepsilon_{i}=0$ and upper bound $\delta_{i}=0.20$, for investment on each stock.

The generated initial portfolio is given in the second column of Table 1.

When model (23) is used to test and to adjust the initial portfolio at time $t=104$, the expected return rate vector $\bar{r}$ is estimated from $r^{t}, t=53,54, \ldots, 104$, and the other parameter values such as $\theta, d_{i}, \varepsilon_{i}$, and $\delta_{i}$ in the model are kept unchanged. Since problem (23) is a linear programming, it is also solved using the "linprog" function in MATLAB.

3.3. Results and Analysis. Test results obtained by model (23) show that the initial portfolio is not optimal at time $t=$ 104 , and it is necessary to adjust the portfolio. Table 1 gives adjusted portfolio for different levels of requirement specified by the value of $\alpha$. The second column gives the initial optimal portfolio at time $t=52$. The values are the investment amounts (\$) on each stock, and the total wealth of the initial portfolio is $\$ 100000000$. The expected wealth of the portfolio after one year is $\$ 113811773$ and the CVaR risk of the portfolio is $\$ 12807285$.

The initial portfolio will be held for one year (until at $t=104)$. The third column gives the value of the portfolio on each stock before rebalance at $t=104$. It can be seen from the results in this column that the total wealth is $\$ 100425613$ before rebalance. If the investor holds the portfolio without any change, then the expected wealth of the portfolio is $\$ 104321125$, and its CVaR risk is $\$ 17050812$.

The fourth column to sixth column list final portfolios $s+x$ generated using model (23) with different values of $\alpha$. It can be seen from column 4 that after rebalancing the total wealth is reduced to $\$ 100206042$ after rebalance, because of the transaction cost, but the expected wealth of the portfolio is increased and the CVaR risk of the portfolio is greatly reduced.

Figure 1 gives the $\alpha$-CVaR curves for some values of $d_{i}^{B}$ (or $d_{i}^{S}$ ), $i=1,2, \ldots, n$, where the vertical axis denotes the requirements of expected returns of the portfolio specified by the value of $\alpha$ in (23), the horizontal axis denotes the corresponding CVaR risk of the portfolio, and $(s+x)$ is final portfolio after rebalancing. It can be observed from the figure that the $\alpha-(\mathrm{CVaR})$ is similar to efficient frontiers in portfolio selection and that the value of $\mathrm{CVaR}$ increases along with the requirement raising in expected return. Moreover, the larger the $d^{B}$ is, the higher the curve is. When $d^{B}$ become larger, the investor will endure larger risk (CVaR) for the same requirement of the expected excess return. This can be understood from another point of view; larger transaction costs will lead to smaller excess return.

\section{Conclusion}

A new portfolio rebalancing model is proposed. The model is different from the existing models due to the constraint for evaluating rebalance feasibility. The constraint is constructed by considering the amount in transaction and the transaction cost. Computational tests based on an initial portfolio are made for different requirements of expected returns. Tests show that the introduced constraint effectively controls the rebalance of the portfolio, and when the rebalance is necessary, the proposed model gives an optimal portfolio which satisfy the investor's requirement and give some guidance for the investment behavior in financial market. Since the proposed model is based on the condition of single portfolio 
TABLE 1: Results for initial portfolio and final portfolio (unit: \$).

\begin{tabular}{|c|c|c|c|c|c|}
\hline Stock number list & $\begin{array}{l}\text { Initial portfolio } \\
\quad \text { at } t=52\end{array}$ & $\begin{array}{l}\text { Portfolio before } \\
\text { rebalance } \\
\text { at } t=104\end{array}$ & $\begin{array}{l}\text { Final portfolio } \\
\quad(s+x) \text { for } \\
\alpha=0.01 \sum_{i=1}^{n} s_{i}\end{array}$ & $\begin{array}{l}\text { Final portfolio } \\
\quad(s+x) \text { for } \\
\alpha=0.02 \sum_{i=1}^{n} s_{i}\end{array}$ & $\begin{array}{l}\text { Final portfolio } \\
\qquad(s+x) \text { for } \\
\alpha=0.03 \sum_{i=1}^{n} s_{i}\end{array}$ \\
\hline 1 & 0 & 0 & 5820477 & 5845410 & 5855215 \\
\hline 2 & 0 & 0 & 6151855 & 7628376 & 9147649 \\
\hline 3 & 0 & 0 & 5657514 & 4682076 & 4062493 \\
\hline 4 & 1907243 & 1803596 & 0 & 0 & 0 \\
\hline 5 & 667971 & 713507 & 7866669 & 8157215 & 8420833 \\
\hline 6 & 1702319 & 1524101 & 14291614 & 14119415 & 13967351 \\
\hline 7 & 10222692 & 9770488 & 20085123 & 19774512 & 19371497 \\
\hline 8 & 13732542 & 10152372 & 0 & 0 & 0 \\
\hline 9 & 18972734 & 19675658 & 20085123 & 20085123 & 20085123 \\
\hline 10 & 0 & 0 & 2967647 & 1938595 & 1020952 \\
\hline 11 & 0 & 0 & 0 & 0 & 0 \\
\hline 12 & 20000000 & 18535111 & 0 & 0 & 0 \\
\hline 13 & 20000000 & 21298069 & 5005473 & 3337153 & 1507138 \\
\hline 14 & 0 & 0 & 3540840 & 4543087 & 5314192 \\
\hline 15 & 12794498 & 16952710 & 8733708 & 10093850 & 11450478 \\
\hline Total wealth & 100000000 & 100425613 & 100206042 & 100204812 & 100202922 \\
\hline Expected wealth & 113811773 & 104321125 & 105325381 & 106329637 & 107333893 \\
\hline CVaR & 12807285 & 17050812 & 7433953 & 7809457 & 8188147 \\
\hline Total transaction costs & - & - & 219572 & 220802 & 222692 \\
\hline
\end{tabular}

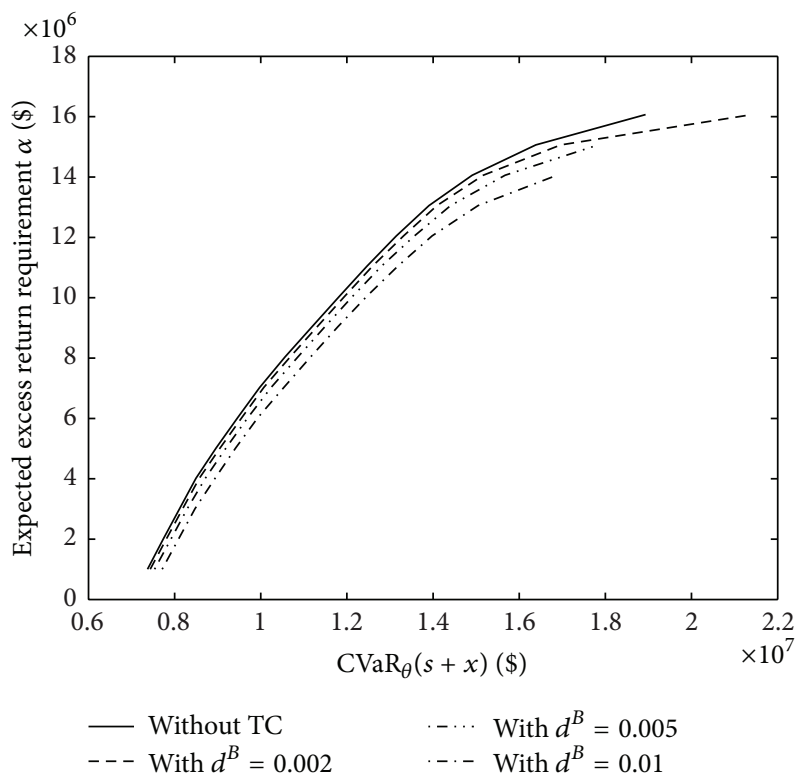

FIGURE 1: Return requirement $\alpha$-CVaR curve with and without transaction costs.

rebalancing, application of the new research into dynamic portfolio rebalancing problem would have significant practical value.

\section{Conflict of Interests}

The authors declare that there is no conflict of interests regarding the publication of this paper.

\section{Acknowledgments}

This work is supported by China Postdoctoral Science Foundation 2013M530418, National Natural Science Foundations of China 71171158 and 11101325.

\section{References}

[1] H. Konno and A. Wijayanayake, "Mean-absolute deviation portfolio optimization model under transaction costs," Journal of the Operations Research Society of Japan, vol. 42, no. 4, pp. 422-435, 1999.

[2] E. Jouini and H. Kallal, "Martingales and arbitrage in securities markets with transaction costs," Journal of Economic Theory, vol. 66, no. 1, pp. 178-197, 1995.

[3] R. D. Arnott and W. H. Wagner, "The measurement and control of trading costs," Financial Analysts Journal, vol. 46, pp. 73-80, 1990.

[4] A. Yoshimoto, "The mean-variance approach to portfolio optimization subject to transaction costs," Journal of the Operations Research Society of Japan, vol. 39, no. 1, pp. 99-117, 1996. 
[5] H. Konno and R. Yamamoto, "Minimal concave cost rebalance of a portfolio to the efficient frontier," Mathematical Programming B, vol. 97, no. 3, pp. 571-585, 2003.

[6] M. S. Lobo, M. Fazel, and S. Boyd, "Portfolio optimization with linear and fixed transaction costs," Technical Report, Department of Electrical Engineering, Stanford University, Stanford, Calif, USA, 2002.

[7] M. S. Lobo, M. Fazel, and S. Boyd, "Portfolio optimization with linear and fixed transaction costs," Annals of Operations Research, vol. 152, pp. 341-365, 2007.

[8] M. J. Best and J. Hlouskova, "Portfolio selection and transactions costs," Computational Optimization and Applications, vol. 24, no. 1, pp. 95-116, 2003.

[9] M. J. Best and J. Hlouskova, "An algorithm for portfolio optimization with transaction costs," Management Science, vol. 51, no. 11, pp. 1676-1688, 2005.

[10] J. E. Mitchell and S. Braun, "Rebalancing an investment portfolio in the presence of convex transaction costs, including market impact costs," Optimization Methods \& Software, vol. 28, no. 3, pp. 523-542, 2013.

[11] M. J. Best and J. Hlouskova, "Quadratic programming with transaction costs," Computers \& Operations Research, vol. 35, no. 1, pp. 18-33, 2008.

[12] W. Zhong, "Portfolio optimization under entropic risk management," Acta Mathematica Sinica, vol. 25, no. 7, pp. 1113-1130, 2009.

[13] P. Artzner, F. Delbaen, J.-M. Eber, and D. Heath, "Coherent measures of risk," Mathematical Finance. An International Journal of Mathematics, Statistics and Financial Economics, vol. 9, no. 3, pp. 203-228, 1999.

[14] R. Tyrrell Rockafellar and S. Uryasex, "Optimization of conditional value-at- risk," Journal of Risk, vol. 2, pp. 21-41, 2000.

[15] R. T. Rockafellar and S. Uryasev, "Conditional value-at-risk for general loss distributions," Journal of Banking and Finance, vol. 26, no. 7, pp. 1443-1471, 2002.

[16] A. Balbás, B. Balbás, and R. Balbás, "CAPM and APT-like models with risk measures," Journal of Banking and Finance, vol. 34, no. 6, pp. 1166-1174, 2010.

[17] C. Roos, T. Terlaky, and J.-Ph. Vial, Theory and Algorithm for Linear Optimization: An Interior Point Approach, John Wiley \& Sons, New York, NY, USA, 1986.

[18] Y. Y. Ye, Interior Point Algorithms: Theory and Analysis, John Wiley \& Sons, New York, NY, USA, 1997.

[19] J. E. Beasley, N. Meade, and T.-J. Chang, "An evolutionary heuristic for the index tracking problem," European Journal of Operational Research, vol. 148, no. 3, pp. 621-643, 2003. 


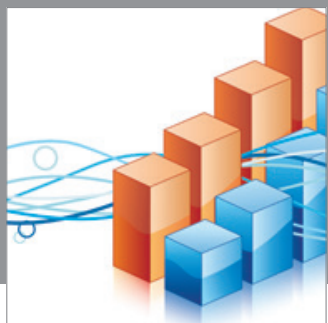

Advances in

Operations Research

mansans

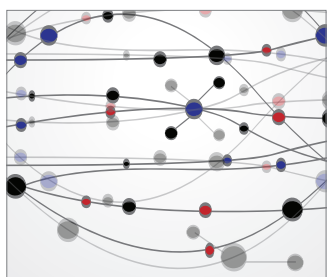

The Scientific World Journal
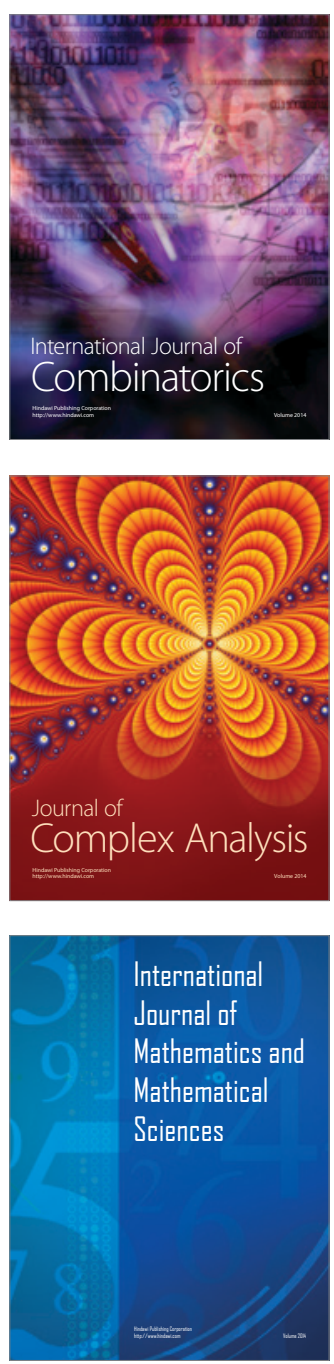
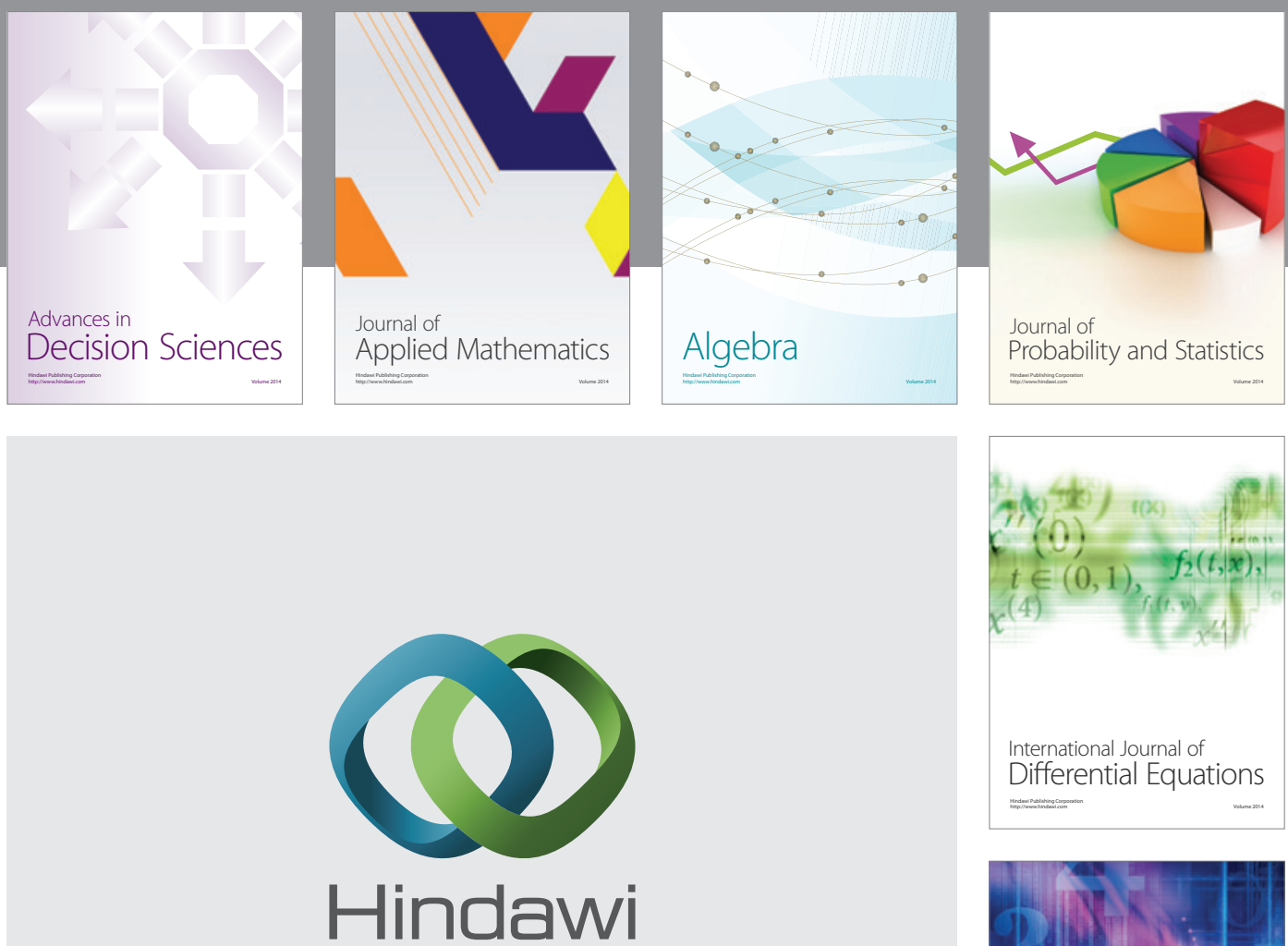

Submit your manuscripts at http://www.hindawi.com
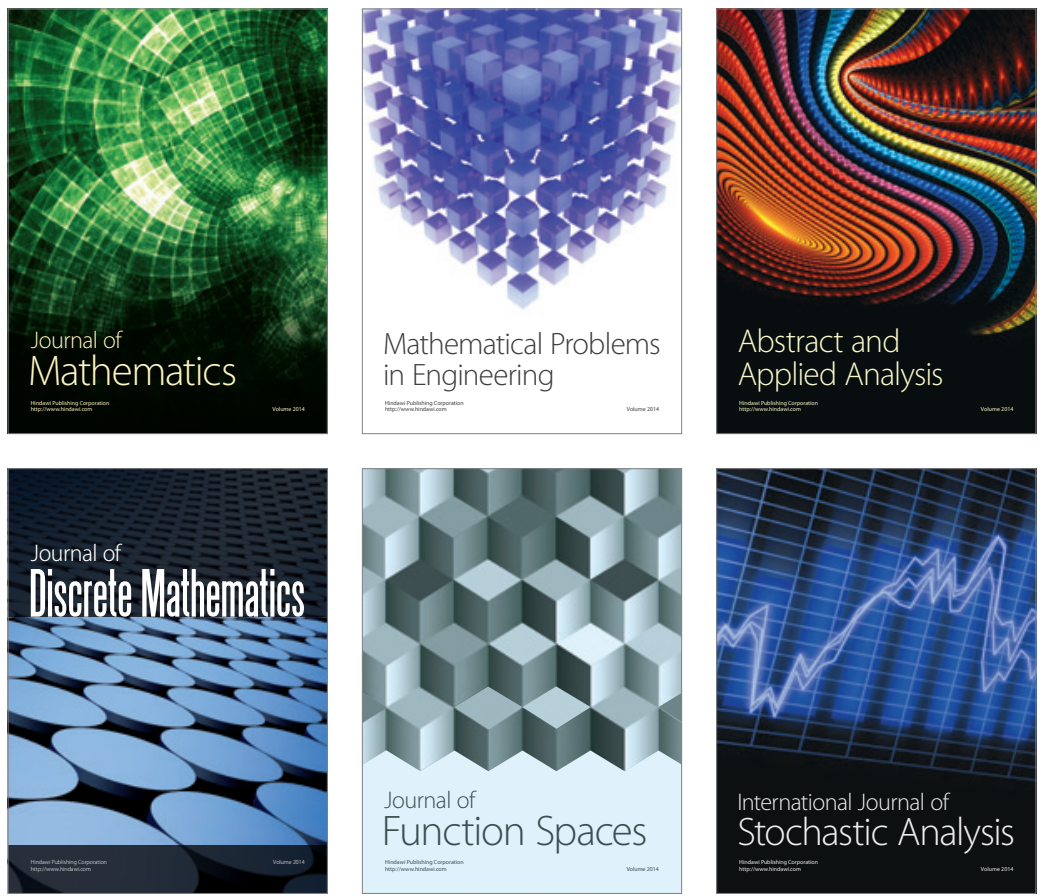

Journal of

Function Spaces

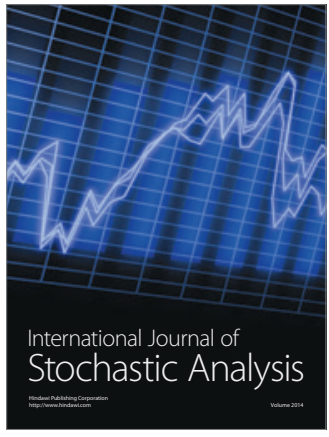

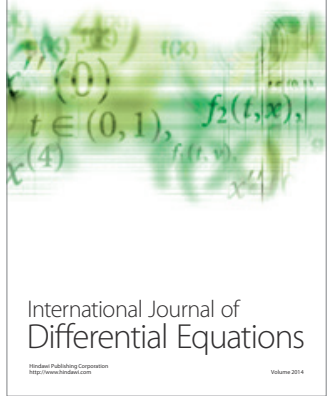
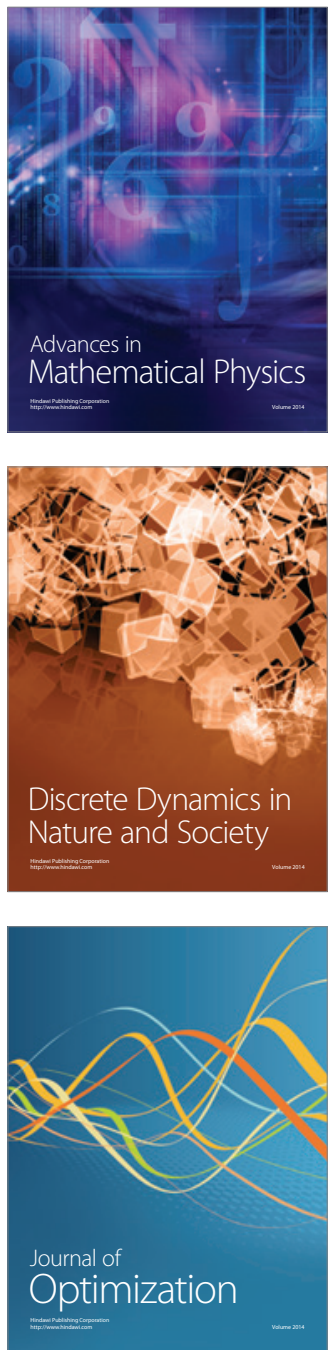